\title{
Detection of virulence factors, phylogroups, serogroups and biofilm formation among $C T X-M-1$ positive Escherichia coli isolated from patients with pyelonephritis.
}

\author{
Seyyed Khalil Shokouhi Mostafavi ${ }^{1}$, Shahin Najar-Peerayeh ${ }^{*}$, Ashraf Mohabbati Mobarez ${ }^{1}$, Mehdi \\ Kardoust Parizi ${ }^{2}$ \\ ${ }^{1}$ Department of Bacteriology, Tarbiat Modares University, Tehran, Iran \\ ${ }^{2}$ Department of Urology, Shariati Hospital, Tehran University of Medical Sciences, Tehran, Iran
}

\begin{abstract}
Objectives: Urinary tract infections are among the most frequent infections caused by Escherichia coli isolates. The aims of this study were determination of antibiotic susceptibility and detection of virulence genes, phylogroups, serogroups and biofilm formation of $E$. coli isolates from patients with pyelonephritis.

Methods: A total of $20 \mathrm{E}$. coli isolates were isolated from pyelonephritis. The antimicrobial susceptibility test was performed for eleven antibiotic disks. The biofilm formation assay was performed with Microtitre Tissue Plate (MTP) assay. The CTX-M1 gene and virulence genes including fimH, fyuA, traT, iutA, papII, kpsMII, ompT, ibeA, sfa, iroN, iucD, afaC, papI and papIII and also phylogroups and serogroups were detected with specific primers.

Results: In the biofilm assay, one isolate produced strong biofilm. Among virulence encoding genes, 19 (95\%) isolates amplified the all fimH, fyuA and traT genes, followed by iutA $(90 \%, \mathrm{n}=18)$, papII (75\%, $\mathrm{n}=17)$, kpsMII $(60 \%, \mathrm{n}=12)$, ompT $(55 \%, \mathrm{n}=11)$, ibeA $(30 \%, \mathrm{n}=6)$, sfa $(20 \%, \mathrm{n}=5)$, and iroN $=$ iuc $D=a f a C=15 \%,(\mathrm{n}=3)$, but none for $\mathrm{papI}$ and $\mathrm{papII}$. The majority $(50 \%, \mathrm{n}=10)$ of $E$. coli isolates from pyelonephritis belonged to the phylogroup B2, followed by phylogroups $D$ and $A$ (each equal to $20 \%, n=4)$ and $B 1(10 \%, n=2)$. Seroproups included $025(20 \%, n=4), 01(15 \%, n=3), 04=018=075$ (each equal to $10 \%, \mathrm{n}=2)$, and $02=012=015=016=1(0.05 \%)$.

Conclusion: The adhesive virulence factors play a critical role in the pathogenesis of pyelonephritis as their prevalence was high. Continued and vigilant surveillance is necessary to monitor the dissemination of antimicrobial resistance in uropathogens.
\end{abstract}

Keywords: Escherichia coli, Biofilms, Virulence, Phylogroups, Serogroups.

Accepted on February 5, 2018

\section{Introduction}

The etiologic agents causing pyelonephritis are narrow and the knowledge of their antimicrobial resistance profile is uncompleted. Urinary Tract Infection (UTI), is among the most frequent bacterial infections, and usually occurs by $E$. coli [1-4]. The pathogenesis of E. coli from UTI has been poorly understood despite profound study. E. coli strains mostly infect the host via entrance through gastrointestinal or vaginal routes. The virulence and also most prevalent clones of $E$. coli representing the infection within a host have not been fully elucidated and possibly the predominant strain tends to be the host's most prevalent fecal strain [3-6].

The ESBL-producing E. coli is isolated from pyelonephritis from hospitalized or out-patients have increasingly posed significant treatment challenges [4-6]. Resistance to the last resort antibiotics (such as carbapenems) is prone to the prolonged hospital stay (occurred in both healthcare and community settings), and history of misuse or overuse drug consumption which leads to increasing morbidity and mortality and costs in health care settings [7,8]. Following surveillance programs to track the increase in the antimicrobial resistance of certain pathogens to fulfil the appropriate strategies for their control is helpful in this regard.

In particular, the spread of ESBL-producing Enterobacteriacea is difficult to restrict at a wider international scale level, due to improper detection of ESBLs and disparity in their reporting [9]. The enhancement in the rate of Multi Drug Resistant (MDR) and ESBL-producing E. coli collected from patients and also environment throughout the globe is a great concern [10]. The aims of this study were detection of antibiotic resistance and virulence factors of $E$. coli isolates from pyelonephritis. Uropathogenic $\mathrm{O} 25 \mathrm{E}$. coli pathogens belong to international clone ST131 which generally contain $C T X-M-1$ 
gene $[11,12]$. UPEC isolates are widespread in the globe and CTX-M-producing strains have been reported increasingly mainly due to the worldwide use of antibiotics. TEM-1, OXA-1 and $a a c\left(6^{\prime}\right)-I b-c r$ are other encoding genes carried by these strains $[13,14]$. The aims of this study were determination of antibiotic susceptibility and detection of virulence genes, phylogroups, serogroups and biofilm formation of $E$. coli isolates from patients with pyelonephritis.

\section{Materials and Methods}

\section{Antibiotic susceptibility testing}

From March 2016 through January 2017, a total of 20 E. coli isolates were isolated from pyelonephritis. The antimicrobial susceptibility test was performed according to the instructions by Clinical and Laboratory Standards Institute (CLSI) version 2016. Eleven antibiotic disks including ampicillin $(10 \mu \mathrm{g})$, cefazolin $(30 \mu \mathrm{g})$, amoxicillin-clavulanic acid $(20 / 10 \mu \mathrm{g})$, ceftazidime $(25 \mu \mathrm{g})$, cefotaxime $(30 \mu \mathrm{g})$, co-trimoxazole $(25$ $\mu \mathrm{g})$, ciprofloxacin $(5 \mu \mathrm{g})$, fosfomycin $(50 \mu \mathrm{g})$, imipenem $(10$ $\mu \mathrm{g})$, gentamicin $(10 \mu \mathrm{g})$ and nitrofurantoin $(100 \mu \mathrm{g})$ were tested [15].

\section{Detection of CTX-M-1 and virulence genes by PCR}

The $C T X-M-1$ and virulence genes including fim $H$, fyuA, traT, iutA, kpsMII, papII, ompT, ibeA, sfa, iroN, iucD, afaC, papI and papIII were detected with specific primers shown in Table 1.

\section{Detection of phylogroups and serogroups}

The specific primers for the amplification of genes for detection of phylogroups and serogroups have been shown in Table 2.

\section{Biofilm formation}

The biofilm formation assay was performed with Microtitre Tissue Plate (MTP) assay. Briefly, bacterial isolates were cultured in Luria-Bertani broth for an overnight and next diluted 1:100 in saline and $20 \mu \mathrm{l}$ of this was inoculated in 180 $\mu \mathrm{l}$ LB broth in the 96-well plate in foursome and incubated for an overnight. The wells of plate were washed with sterile water and then the crystal violet was added and kept in ambient temperature for $15 \mathrm{~min}$. the wells were washed and methanol was added for the fixation and left to dried. Next, the ethanol was added and the solvent biofilm opacity was measured at $\mathrm{OD}=540 \mathrm{~nm}$ and $620 \mathrm{~nm}$ (negative control) with ELISA reader [16].

\section{Data analysis}

Data was analysed using SPSS software version 20, with ANOVA test and t-test and 95\% confidence interval $(\mathrm{p}<0.05$ being significant result).

\section{Results}

\section{Patients' demographic data}

Twenty patients included 14 females and 6 males with the mean ages of 54 and 66 years, respectively and mostly because of urinary tract infection $(70 \%, \mathrm{n}=14)$, followed by kidney impair $(15 \%, n=3)$, leukemia $(10 \%, n=2)$ and bladder stone $(5 \%, n=1)$. They were all inpatients in the hospital wards including 6 from urology, 7 from emergency and 7 from each of kidney implantation, blood emergency, nephrology, internal ICU, and internal general wards.

\section{The antibiotic susceptibility test}

All $20 C T X-M-1$ positive $E$. coli isolates from pyelonephritis were susceptible to imipenem, meropenem and fosfomycin and all of them were resistant to ceftazidime, amoxicillin and erythromycin (Table 3 ).

\section{The virulence genes}

Among virulence encoding genes, 19 (95\%) isolates amplified the all fimH, fyuA and traT genes, followed by iutA $(90 \%$, $\mathrm{n}=18)$, papII $(75 \%, \mathrm{n}=17), \operatorname{kpsMII}(60 \%, \mathrm{n}=12)$, ompT $(55 \%$, $\mathrm{n}=11)$, ibeA $(30 \%, \quad \mathrm{n}=6), \quad s f a \quad(20 \%, \quad \mathrm{n}=5), \quad$ and iroN $=i u c D=a f a C=15 \%,(\mathrm{n}=3)$, but none for $p a p I$ and $p a p I I$.

\section{The phylogenetic grouping and serogrouping}

The majority $(50 \%, \mathrm{n}=10)$ of $E$. coli isolates from pyelonephritis belonged to the phylogroup B2, followed by phylogroups D and A (each equal to $20 \%, \mathrm{n}=4)$ and B1 $(10 \%$, $\mathrm{n}=2)$ which is shown in Table 4. Serogroups included O25 $(20 \%, \mathrm{n}=4), \mathrm{O} 1(15 \%, \mathrm{n}=3), \mathrm{O} 4=\mathrm{O} 18=\mathrm{O} 75$ (each equal to $10 \%, \mathrm{n}=2)$, and $\mathrm{O} 2=\mathrm{O} 12=\mathrm{O} 15=\mathrm{O} 16=1(0.05 \%)$, but two of them were non-type able with this method. Among 10 isolates belonged to B2 phylogroup, all were iutA, trat $T$ and fimH positive and 9 of them were $f y u A$ and moreover the major serogroups in this phylogroup were $\mathrm{O} 75(\mathrm{n}=2)$, followed by one isolate belonging to each $\mathrm{O} 12, \mathrm{O} 18, \mathrm{O} 25, \mathrm{O} 4, \mathrm{O} 1$ and $\mathrm{O} 15$, and two isolates were not typed by this method. There was no significant relation between phylogroups and serogroups among UPEC isolates. Among four isolates belonging to each phylogroups A and D, serogroups, O25 and O1 ( $\mathrm{n}=2,50 \%)$ were mostly detected respectively (Table 4).

\section{Biofilm formation}

In the biofilm assay, one isolate produced strong biofilm, four isolates produced moderate and 8 isolates weak biofilm, but 7 isolates produced no biofilm (Table 5).

Table 1. The specific primers used for detection of virulence genes.

\begin{tabular}{lll}
\hline Primer & Sequence (5' to 3') & Amplicon (bp) \\
\hline CTX-M1 & F-GGTTAAAAAATCACTGCGTC & 863 \\
\cline { 2 - 3 } & R-TTGGTGACGATTTTCGCCGC & \\
\hline
\end{tabular}


Detection of virulence factors, phylogroups, serogroups and biofilm formation among CTX-M-1 positive Escherichia coli isolated from patients with pyelonephritis

\begin{tabular}{|c|c|c|}
\hline \multirow[t]{2}{*}{ fimH } & F: TGCAGAACGGATAAGCCGTGG & 508 \\
\hline & \multicolumn{2}{|l|}{ R: CTCCGGAGAACTGGGTGCATCTTAC } \\
\hline \multirow[t]{2}{*}{ fyuA } & F: GCGACGGGAAGCGATGACTTA & 774 \\
\hline & R: CGCAGTAGGCACGATGTTGTA & \\
\hline \multirow[t]{2}{*}{$\operatorname{traT}$} & F: GCGCATTTGCTGATACTGTTG & 429 \\
\hline & R: CATCCAGACGATAAGCATGAGCA & \\
\hline \multirow[t]{2}{*}{ iutA } & F: GCGCGTAGCCGATGAAAT & 302 \\
\hline & R: CACTGAAAACAAGATTGAT & \\
\hline \multirow[t]{2}{*}{ kpsMII } & F: AAGTCAAAGCAGGGGTTGCCCG & 668 \\
\hline & R: GACGCCGACATTAAGACGCAG & \\
\hline \multirow[t]{2}{*}{ ompT } & F: ATCTAGCCGAAGAAGGAGGC & 559 \\
\hline & R: CCCGGGTCATAGTGTTCATC & \\
\hline \multirow[t]{2}{*}{$i b e A$} & F: AGGCAGGTGTGCGCCGCGTAC & 170 \\
\hline & R: TGGTGCTCCGGCAAACCATGC & \\
\hline
\end{tabular}

\begin{tabular}{|c|c|c|}
\hline \multirow[t]{2}{*}{ sfa } & F: CTCCGGAGAACTGGGTGCATCTTAC & 408 \\
\hline & R: CGGAGGAGTAATTACAAACCTGGCA & \\
\hline \multirow[t]{2}{*}{ iroN } & F: AAGTCAAAGCAGGGGTTGCCCG & 668 \\
\hline & R: GACGCCGACATTAAGACGCAG & \\
\hline \multirow[t]{2}{*}{$i u c D$} & F: TACCGGATTGTCATATGCAGACCGT & 602 \\
\hline & R: AATATCTTCCTCCAGTCCGGAGAAG & \\
\hline \multirow[t]{2}{*}{$a f a c$} & F: TAAGGAAGTGAAGGAGCGTG & 802 \\
\hline & R: CCAGTAACTGTCCGTGACA & \\
\hline \multirow[t]{2}{*}{ papGl } & F: TCGTGCTCAGGTCCGGAATTT & 461 \\
\hline & R: TGGCATCCCCCAACATTATCG & \\
\hline \multirow[t]{2}{*}{ papGII } & F: GGGATGAGCGGGCCTTTGAT & 190 \\
\hline & R: CGGGCCCCCAAGTAACTCG & \\
\hline \multirow[t]{2}{*}{ раpGIII } & F: GGCCTGCAATGGATTTACCTGG & 258 \\
\hline & R: CCACCAAATGACCATGCCAGAC & \\
\hline
\end{tabular}

Table 2. The specific primer for the detection of serogroups.

\begin{tabular}{|c|c|c|c|}
\hline Target gene & Sequence (5' to $\left.3^{\prime}\right)$ & Amplicon Size, bp & Optimal annealing temperature $\left({ }^{\circ} \mathrm{C}\right)$ \\
\hline \multirow[t]{2}{*}{$r f b 01$} & F: ATACCGACGACGCCGATCTG & 189 & 59 \\
\hline & R: CCAGAAATACACTTGGAGAC & & \\
\hline \multirow[t]{2}{*}{ rfbO2 } & F: ATACCGACGACGCCGATCTG & 274 & 59 \\
\hline & R: GTGACTATTTCGTTACAAGC & & \\
\hline \multirow[t]{2}{*}{$r f b 018$} & F: ATACCGACGACGCCGATCTG & 360 & 59 \\
\hline & R: GAAGATGGCTATAATGGTTG & & \\
\hline \multirow[t]{2}{*}{ rfbO16 } & F: ATACCGACGACGCCGATCTG & 450 & 59 \\
\hline & R: GGATCATTTATGCTGGTACG & & \\
\hline \multirow[t]{2}{*}{$r f b O 6$} & F: ATACCGACGACGCCGATCTG & 584 & 59 \\
\hline & R: AAATGAGCGCCCACCATTAC & & \\
\hline \multirow[t]{2}{*}{$r f b O 7$} & F: ATACCGACGACGCCGATCTG & 722 & 59 \\
\hline & R: CGAAGATCATCCACGATCCG & & \\
\hline \multirow[t]{2}{*}{ rfbO4 } & F: ATACCGACGACGCCGATCTG & 193 & 67 \\
\hline & R: AGGGGCCATTTGACCCACTC & & \\
\hline \multirow[t]{2}{*}{ rfbO12 } & F: ATACCGACGACGCCGATCTG & 239 & 59 \\
\hline & R: GTGTCAAATGCCTGTCACCG & & \\
\hline \multirow[t]{2}{*}{$r f b O 25$} & F: ATACCGACGACGCCGATCTG & 313 & 59 \\
\hline & R: GAGATCCAAAAACAGTTTGTG & & \\
\hline \multirow[t]{2}{*}{ rfbO75 } & F: ATACCGACGACGCCGATCTG & 419 & 58 \\
\hline & R: GTAATAATGCTTGCGAAACC & & \\
\hline \multirow[t]{2}{*}{ rfbO15 } & F: ATACCGACGACGCCGATCTG & 536 & 59 \\
\hline & R: TGATAATGACCAACTCGACG & & \\
\hline
\end{tabular}




\begin{tabular}{|c|c|c|c|}
\hline \multirow[t]{2}{*}{ rfbO157 } & F: ATACCGACGACGCCGATCTG & 672 & 59 \\
\hline & R: TACGACAGAGAGTGTCTGAG & & \\
\hline \multirow[t]{2}{*}{ chuA } & F: GACGAACCAACGGTCAGGAT & 279 & 54 \\
\hline & R: TGCCGCCAGTACCAAAGACA & & \\
\hline \multirow[t]{2}{*}{$y j a A$} & F: TGAAGTGTCAGGAGACGCTG & 211 & 54 \\
\hline & R: ATGGAGAATGCGTTCCTCAAC & & \\
\hline \multirow[t]{2}{*}{ tspE4C2 } & F: GAGTAATGTCGGGGCATTCA & 152 & 54 \\
\hline & R: CGCGCCAACAAAGTATTACG & & \\
\hline
\end{tabular}

Table 3. The antibiotic susceptibility of 20 E. coli isolates from pyelonephritis.

\begin{tabular}{|c|c|c|c|c|c|c|c|c|c|c|c|c|c|c|}
\hline Antibiotic & & FO & PTZ & CAZ & CTX & AMX & CZ & AMC & IMI & FM & GM & CIP & MEN & SXT \\
\hline $\begin{array}{l}\text { Susceptible } \\
\text { (\%) }\end{array}$ & no & $20(100)$ & $19(95)$ & $2(10)$ & - & - & - & $2(10)$ & $20(100)$ & $20(100)$ & $7(35)$ & $5(25)$ & $20(100)$ & $15(75)$ \\
\hline $\begin{array}{l}\text { Resistant } \\
(\%)\end{array}$ & No & - & $1(5)$ & $18(90)$ & $20(100)$ & $20(100)$ & $20(100)$ & $18(80)$ & - & - & $13(65)$ & $15(75)$ & - & $5(25)$ \\
\hline
\end{tabular}

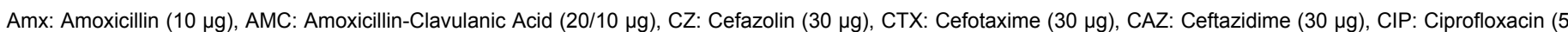

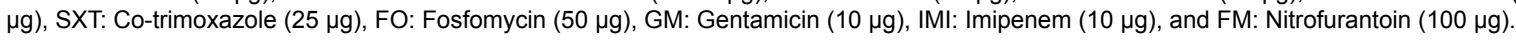

Table 4. The phylogroups, serogroups and virulence factors of UPEC in this study.

\begin{tabular}{|c|c|c|c|c|c|c|c|c|c|c|c|c|c|c|c|c|c|}
\hline Isolate & Phylogroup & Serotype & papl & papll & paplll & ompT & $s f a$ & iroN & $i u c D$ & fyuA & $a f a C$ & fimH & $i b e A$ & $\operatorname{traT}$ & kpsMII & iutA & Biofilm \\
\hline 1 & $\mathrm{D}$ & O1 & - & + & - & + & - & - & - & + & - & + & - & + & + & + & M \\
\hline 2 & $A$ & $\mathrm{Nd}$ & - & + & - & + & - & - & - & + & - & + & - & + & - & - & $\mathrm{N}$ \\
\hline 3 & B2 & O18 & - & - & - & - & - & - & - & + & - & + & + & + & - & + & M \\
\hline 4 & B2 & $\mathrm{Nd}$ & - & - & - & - & - & - & - & - & + & + & + & + & - & + & $\mathrm{N}$ \\
\hline 5 & B1 & $\mathrm{O} 25$ & - & + & - & + & - & - & + & + & - & + & - & + & + & + & $S$ \\
\hline 6 & $A$ & $\mathrm{O} 25$ & - & + & - & - & - & - & - & + & - & + & + & + & - & + & W \\
\hline 7 & B2 & O12 & - & + & - & - & + & - & - & + & - & + & - & + & - & + & $\mathrm{N}$ \\
\hline 8 & B2 & O15 & - & + & - & - & - & - & - & + & - & + & - & + & - & + & W \\
\hline 9 & A & $\mathrm{O} 2$ & - & + & - & - & + & - & - & + & - & - & + & + & - & + & $\mathrm{N}$ \\
\hline 10 & B2 & O1 & - & - & - & + & - & - & - & + & - & + & - & + & + & + & W \\
\hline 11 & $D$ & O18 & - & + & - & - & + & + & - & + & - & + & - & + & + & - & W \\
\hline 12 & B2 & $\mathrm{O} 75$ & - & + & - & + & + & + & - & + & - & + & + & + & + & + & W \\
\hline 13 & B2 & $\mathrm{O} 4$ & - & + & - & + & + & - & - & + & + & + & - & + & + & + & W \\
\hline 14 & A & O16 & - & + & - & + & - & - & - & + & - & + & - & + & + & + & $\mathrm{N}$ \\
\hline 15 & B1 & $\mathrm{O} 25$ & - & + & - & - & - & - & - & + & - & + & - & + & + & + & $\mathrm{N}$ \\
\hline 16 & B2 & O75 & - & + & - & + & - & - & + & + & - & + & + & + & + & + & W \\
\hline 17 & B2 & O25 & - & + & - & + & - & - & + & + & - & + & - & + & + & + & $M$ \\
\hline 18 & B2 & $\mathrm{Nd}$ & - & + & - & + & - & - & - & + & - & + & - & + & - & + & $\mathrm{N}$ \\
\hline 19 & $D$ & O4 & - & + & - & - & - & - & - & + & + & + & - & + & + & + & $\mathrm{N}$ \\
\hline 20 & $D$ & 01 & - & + & - & + & - & + & - & + & - & + & - & - & + & + & W \\
\hline
\end{tabular}

In this table Nd: Non detect; S: Strong; M: Moderate; W: Weak; N: Negative. 
Table 5. The biofilm formation among UPEC isolates, ODc: OD of control.

\begin{tabular}{lll}
\hline Biofilm level & OD cut off & No (\%) \\
\hline Strong & OD $>4 \times$ ODc & $1(5)$ \\
\hline Moderate & $2 \times$ ODc $<O D \leq 4 \times$ ODc & $4(20)$ \\
\hline Weak & ODc $<$ OD $\leq 2 \times$ ODc & $8(40)$ \\
\hline No biofilm & OD $\leq 0.08324$ & $7(35)$ \\
\hline
\end{tabular}

\section{Discussion}

Healthcare associated UTI is associated with higher therapy failure and thus patients should be more under care to avoid morbidity [17,18]. In this study, three patients had kidney failure which developed pyelonephritis with the CTX-M1 producing UPEC (ANOVA test, $\mathrm{p}>0.05$ ). Furthermore, 20/95 of CTX-M1 producing UPEC isolates had caused this type of infection which was included in this study. Previous studies have exhibited that the rate of ESBL-producing E. coli has increased in Iran [19-21]. CTX-M-1 producing isolates are predominant in the Middle East region, Eastern Europe, western areas of Russia, India, United States and Australia. We found that potential risk factors for spread of pyelonephritis caused by $C T X-M-1$ producing $E$. coli were urinary tract infection $(70 \%, \mathrm{n}=14)$, followed by kidney impair $(15 \%, \mathrm{n}=3)$, leukemia $(10 \%, \mathrm{n}=2)$ and bladder stone $(5 \%, \mathrm{n}=1)$ which are approximately similar to risk factors from previous surveys including prior use of antimicrobials and beta-lactams, prior UTI and hospitalization caused by ceftriaxone-resistant organisms [22,23]. In this study, the rate of fluoroquinolones resistance among $E$. coli isolates from pyelonephritis was $75 \%$ which is a concern. The $C T X-M-1$ enzyme is the predominant encoding gene responsible for ESBL production by $E$. coli isolates worldwide. There is no previous study for the assessment of CTX-M-1 producing E. coli from pyelonephritis in Iran.

Furthermore, using ANOVA test analysis, among virulence genes, $19(95 \%)$ isolates amplified the all fimH, fyuA and traT genes $(\mathrm{p}<0.001)$, followed by iutA $(90 \%, \mathrm{n}=18)(\mathrm{p}<0.001)$, papII $(75 \%, \mathrm{n}=17)(\mathrm{p}=0012)$, kpsMII $(60 \%, \mathrm{n}=12)$, ompT $(55 \%, \mathrm{n}=11)$, ibeA $(30 \%, \mathrm{n}=6), \quad s f a(20 \%, \mathrm{n}=5)$, and iroN $=i u c D=a f a C=15 \%,(\mathrm{n}=3)$, but none for $p a p I$ and papIII. It was found that among fimH, fyuA and traT positive isolates, $14 / 19,12 / 19$ and $13 / 19$ of them were resistant to ciprofloxacin, respectively. Four moderate biofilm producers in this study amplified the all fimH, fyuA and traT genes and three amplified iutA, papII, ompT, kpsMII, sfa and ibeA genes. This result exhibits the role of adhesion genes in the ability of UPEC isolates for biofilm formation. Moreover, 11/19, 13/19 and $12 / 19$ of them were resistant to aminoglycosides. Furthermore, $11 / 18$ and $12 / 18$ of iutA positive isolates were fluoroquinolones and aminoglycoside resistant UPEC.
Similarly, a high number of UPEC isolates from South Korea were fyuA positive [24]. Another study in Mexico showed that kpsMII and fimH genes were high among UPEC isolates similar to this study [25]. In Tunisia, $68 \%$ and $41 \%$ of UPEC were fimH and pap positive [26]. In Jahrom city, the prevalence of $p a p G, a f a C, s f a$, fimH, ibeA, and iucD were $53.3 \%, 51.7 \%, 53.3 \%, 56.7 \%, 20 \%$, and $73.3 \%$, respectively which were mostly similar to findings of the present survey [27]. There was a difference between children and adults regarding the prevalence of hlyA, kpsMII and ibeA among UPEC [28], but this was concluded in our study. Momtaz in Shahrekord found that fim $H$ virulence gene was detected in $86.17 \%$ of UPEC isolates, and $2.43 \%$ of them belonged to the O1, O2, O6, O7 and O16 serogroups [29]. A great number of E. coli virulence factors are encoded by Pathogenicity Islands (PIs) and are known as "Pathogen Associated Molecular Patterns" (PAMPs) [30,31], however the exact prevalence or role of adhesive virulence factors in the pathogenesis of pyelonephritis is not obvious. It was shown that extra-intestinal isolates were more efficient colonizers of the digestive tract compared to non-pathogenic strains [32].

In this study, among 10 isolates belonged to $\mathrm{B} 2$ phylogroup, all were iutA, traT and fimH positive and 9 of them were fyuA positive and moreover the major serogroups in this phylogroup were $\mathrm{O} 75(\mathrm{n}=2)$, followed by one isolate belonging to each $\mathrm{O} 12, \mathrm{O} 18, \mathrm{O} 25, \mathrm{O} 4, \mathrm{O} 1$ and $\mathrm{O} 15$, and two isolates were not typed by this method. There was no significant relation between phylogroups and serogroups among UPEC isolates. Among four isolates belonging to each phylogroups $\mathrm{A}$ and $\mathrm{D}$, serogroups, O25 and O1 (n=2,50\%) were mostly detected respectively. therefore, it is proposed that more isolates are needed to decide surely about the relationships between phylogroups and serogroups of UPEC isolates. Furthermore, one strong biofilm producer was detected in this study and was belonged to the phylogroup B1 and also contained papII, отрT, iисD, fyuA, fimH, traT, kpsMII and iutA genes and exhibited alpha-hemolysis. Moreover, three moderate-biofilm producing isolates were belonged to the $\mathrm{D}(\mathrm{n}=1)$ and $\mathrm{B} 2(\mathrm{n}=2)$ phylogroups. In this study, the phylogroups B2 was predominant among E. coli isolates from pyelonephritis as exhibited in previous studies [33-35]. From our results and scarce previous studies it is concluded that the adhesive factors have an important role in the colonization, biofilm formation and dissemination of strains in the body. There a limitation in this study where we did not assess the expression level of the adhesive or other virulence factors related to the pyelonephritis. Several drawbacks in this study include small sample size and studied patients, and lack of gene expression assay for detected virulence factors.

\section{Conclusion}

The antibiotic resistance rate was high among isolates of $E$. coli from pyelonephritis. The fimH, fyuA and traT genes 
$(\mathrm{p}<0.001)$, followed by iutA $(90 \%, \mathrm{n}=18)(\mathrm{p}<0.001)$, papII $(75 \%, \mathrm{n}=17)(\mathrm{p}=0012)$ and $k p s M I I(60 \%, \mathrm{n}=12)$ adhesive virulence factors was highly detected, possibly exhibiting their role in the pathogenesis of pyelonephritis as their prevalence was high. Furthermore, fluoroquinolones and aminoglycoside resistance was highly determined exhibiting a threat due to the presence of pathogenic and drug-resistant UPEC in pyelonephritis. Continued and vigilant surveillance is necessary for the monitoring the spread of antimicrobial resistance among uropathogens.

\section{Acknowledgments}

This work was supported by the grants provided by Faculty of Medical Sciences, Tarbiat Modares University, Tehran, Iran.

\section{References}

1. Najar Peerayeh S, Eslami M, Memariani M, Siadat SD. High prevalence of blaCTX-M-1 group extended-spectrum $\beta$-lactamase genes in Escherichia coli isolates from Tehran. Jundishapur J Microbiol 2013; 6: 1-6.

2. Hoban DJ, Nicolle LE, Hawser S, Bouchillon S, Badal R. Antimicrobial susceptibility of global inpatient urinary tract isolates of Escherichia coli: results from the Study for Monitoring Antimicrobial Resistance Trends (SMART) program: 2009-2010. Diagn Microbiol Infect Dis 2011; 70: 507-511.

3. Navidinia M, Peerayeh SN, Fallah F, Bakhshi B. Phylogenetic groups and pathogenicity island markers in Escherichia coli isolated from children. Jundishapur J Microbiol 2013; 6.

4. Navidinia M, Peerayeh SN, Fallah F, Bakhshi B, Sajadinia RS. Phylogenetic grouping and pathotypic comparison of urine and fecal Escherichia coli isolates from children with urinary tract infection. Brazil J Microbiol 2014; 45: 509-514.

5. Rodríguez-Bano J, Navarro MD, Romero L, MartínezMartínez L, Muniain MA, Perea EJ. Epidemiology and clinical features of infections caused by extended-spectrum beta-lactamase-producing Escherichia coli in nonhospitalized patients. J Clin Microbiol 2004; 42: 1089-1094.

6. Memariani M, Peerayeh SN, Salehi TZ, Mostafavi SKS. Occurrence of SHV, TEM and CTX-M $\beta$-lactamase genes among enteropathogenic Escherichia coli strains isolated from children with diarrhea. Jundishapur J Microbiol 2015; 8 .

7. Oteo J, Delgado-Iribarren A, Vega D, Bautista V, Rodríguez MC, Velasco M. Emergence of imipenem resistance in clinical Escherichia coli during therapy. Int $\mathrm{J}$ Antimicrob Agents 2008; 32: 534-537.

8. Poirel L, Kieffer N, Liassine N, Thanh D, Nordmann P. Plasmid-mediated carbapenem and colistin resistance in a clinical isolate of Escherichia coli. Lancet Infect Dis 2016; 16.
9. Tangden T, Adler M, Cars O, Sandegren L, Lowdin E. Frequent emergence of porin-deficient subpopulations with reduced carbapenem susceptibility in ESBL-producing Escherichia coli during exposure to ertapenem in an in vitro pharmacokinetic model. J Antimicrob Chemother 2013; 68: 1319-1326.

10. Valenza G, Nickel S, Pfeifer Y, Eller C, Krupa E, LehnerReindl V. Extended-spectrum- $\beta$-lactamase-producing Escherichia coli as intestinal colonizers in the German community. Antimicrob Agents Chemother 2014; 58: 1228-1230.

11. Nicolas-Chanoine MH, Bertrand X, Madec JY. Escherichia coli ST131, an intriguing clonal group. Clin Microbiol Rev 2014; 27: 543-574.

12. Sallem RB, Slama KB, Estepa V, Cheikhna EO, Mohamed AM, Chairat S. Detection of CTX-M-15-producing Escherichia coli isolates of lineages ST410-A, ST617-A and ST354-D in faecal samples of hospitalized patients in a Mauritanian hospital. J Chemother 2015; 27: 114-116.

13. Rodriguez I, Thomas K, Van Essen A, Schink AK, Day M, Chattaway M. Chromosomal location of bla CTX-M genes in clinical isolates of Escherichia coli from Germany, the Netherlands and the UK. Int J Antimicrob Agents 2014; 43: 553-557.

14. Forde BM, Zakour NLB, Stanton-Cook M, Phan M-D, Totsika M, Peters KM. The complete genome sequence of Escherichia coli EC958: a high quality reference sequence for the globally disseminated multidrug resistant E. coli O25b: H4-ST131 clone. PloS One 2014; 9: 104400.

15. Jorgensen JH, Turnidge JD. Susceptibility test methods: dilution and disk diffusion methods. Manual Clin Microbiol Am Soc Microbiol 2015; 1253-1273.

16. Danese PN, Pratt LA, Dove SL, Kolter R. The outer membrane protein, Antigen 43, mediates celltocell interactions within Escherichia coli biofilms. Mol Microbiol 2000; 37: 424-432.

17. Ha YE, Kang CI, Joo EJ, Park SY, Kang SJ, Wi YM. Clinical implications of healthcare-associated infection in patients with community-onset acute pyelonephritis. Scand J Infect Dis 2011; 43: 587-595.

18. Peerayeh SN, Rostami E, Eslami M, Rezaee MA. High frequency of extended-spectrum $\beta$-lactamase-producing Klebsiella pneumoniae and Escherichia coli isolates from male patients urine. Arc Clin Infect Dis 2016; 11.

19. Behrooozi A, Rahbar M, Jalil V. Frequency of extended spectrum beta-lactamase (ESBLs) producing Escherichia coli and Klebseilla pneumonia isolated from urine in an Iranian 1000-bed tertiary care hospital. African J Microbiol Res 2010; 4: 881-884.

20. Rezai MS, Salehifar E, Rafiei A, Langaee T, Rafati M, Shafahi K. Characterization of multidrug resistant extended-spectrum beta-lactamase-producing Escherichia coli among uropathogens of pediatrics in North of Iran. Biomed Res Int 2015; 2015.

21. Haghighatpanah M, Nejad ASM, Mojtahedi A, Amirmozafari N, Zeighami H. Detection of extended- 
spectrum $\beta$-lactamase (ESBL) and plasmid-borne bla CTX$M$ and bla TEM genes among clinical strains of Escherichia coli isolated from patients in the north of Iran. J Glob Antimicrob Resist 2016; 7: 110-113.

22. Bedoin M, Cazorla C, Lucht F, Berthelot P, Boyer M, Carricajo A. Risk factors for quinolone-resistance in women presenting with Escherichia coli acute pyelonephritis. Med et Maladies Infect 2014; 44: 206-216.

23. Van der Starre WE, Van Nieuwkoop C, Paltansing S, Vant Wout JW, Groeneveld GH, Becker MJ. Risk factors for fluoroquinolone-resistant Escherichia coli in adults with community-onset febrile urinary tract infection. J Antimicrob Chemother 2010; 66: 650-656.

24. Yun KW, Kim HY, Park HK, Kim W, Lim IS. Virulence factors of uropathogenic Escherichia coli of urinary tract infections and asymptomatic bacteriuria in children. $\mathrm{J}$ Microbiol Immunol Infect 2014; 47: 455-461.

25. Paniagua-Contreras GL, Monroy-Pérez E, RodríguezMoctezuma JR, Domínguez-Trejo P, Vaca-Paniagua F, Vaca S. Virulence factors, antibiotic resistance phenotypes and O-serogroups of Escherichia coli strains isolated from community-acquired urinary tract infection patients in Mexico. J Microbiol Immunol Infect 2017; 50: 478-485.

26. Tarchouna M, Ferjani A, Ben-Selma W, Boukadida J. Distribution of uropathogenic virulence genes in Escherichia coli isolated from patients with urinary tract infection. Int J Infect Dis 2013; 17: 450-453.

27. Asadi S, Kargar M, Solhjoo K, Najafi A, Ghorbani-Dalini S. The association of virulence determinants of uropathogenic Escherichia coli with antibiotic resistance. Jundishapur J Microbiol 2014; 7.

28. Vollmerhausen T, Katouli M. Molecular characterisation of Escherichia coli isolated from hospitalised children and adults with urinary tract infection. Eur J Clin Microbiol Infect Dis 2014; 33: 975-982.

29. Momtaz H, Karimian A, Madani M, Dehkordi FS, Ranjbar R, Sarshar M. Uropathogenic Escherichia coli in Iran: serogroup distributions, virulence factors and antimicrobial resistance properties. Ann Clin Microbiol Antimicrob 2013; 12: 8 .

30. Lloyd AL, Henderson TA, Vigil PD, Mobley HL. Genomic islands of uropathogenic Escherichia coli contribute to virulence. J Bacteriol 2009; 191: 3469-3481.

31. Lloyd AL, Rasko DA, Mobley HL. Defining genomic islands and uropathogen-specific genes in uropathogenic Escherichia coli. J Bacteriol 2007; 189: 3532-3546.

32. Tourret J, Willing BP, Croxen MA, Dufour N, Dion S, Wachtel S. Small intestine early innate immunity response during intestinal colonization by Escherichia coli depends on its extra-intestinal virulence status. PloS One 2016; 11: 0153034 .

33. Agarwal J, Mishra B, Srivastava S, Srivastava R, Pandey A. Virulence determinants in Escherichia coli associated with recurrent cystitis in sexually active women. Microb Pathogene 2014; 74: 38-41.

34. Ali I, Rafaque Z, Dasti JI, Graham SE, Salzman E, Foxman B. Uropathogenic E. coli from Pakistan have high prevalence of multidrug resistance, ESBL, and O25bST131. Open Forum Infect Dis 2016.

35. da Silva LC, de Mello Santos AC, Silva RM. Uropathogenic Escherichia coli pathogenicity islands and other ExPEC virulence genes may contribute to the genome variability of enteroinvasive E. coli. BMC Microbiol 2017; 17: 68 .

\section{*Correspondence to}

Shahin Najar-Peerayeh

Department of Bacteriology

Tarbiat Modares University

Iran 